\title{
STELLAR POPULATIONS OF DWARF GALAXIES
}

\author{
ELCHANAN ALMOZNINO AND NOAH BROSCH \\ The School of Physics and Astronomy, Raymond and Beverly \\ Sackler Faculty of Exact Sciences, Tel Aviv University, Tel \\ Aviv 69978, Israel
}

We selected a sample of dwarf galaxies in the Virgo cluster from the original catalog of Bingelli, Sandage and Tamman (1985), which have been measured at $21 \mathrm{~cm}$ by Hoffman et al. (1987). The original sample was selected to have both high surface brightness objects (classified as BCD or anything/BCD), and low surface brightness galaxies (classified as ImIII, ImIV or ImV). An additional selection was done on the total amount of HI, as given in Hoffman et al. We selected from each surface brightness group objects with high HI content (flux integral larger than a set threshold), and others with low HI content. We thus defined four samples differing in surface brightness level and total HI content.

The initial idea was to test star formation processes on galaxies devoid of spiral density waves, thus the selection of dwarf galaxies. The two parameters on which to test the star-forming process were selected to be past (but recent) star formation and history of star formation, as evidenced by broad-band colors and tests against star formation and stellar synthesis models, and available material for future star formation, namely total HI content.

The galaxies discussed here are from the high surface brightness group. The observations were done at the Wise Observatory, where broad band $\mathrm{B}$, $\mathrm{V}, \mathrm{R}$ and I images were obtained, as well as $\mathrm{H} \alpha$ line and nearby continuum. Some objects were observed with IUE to obtain their SW low dispersion spectra. A few appeared on UV images of FAUST (Bowyer el al. 1993) and for others it was possible to derive upper limits of the UV flux. Finally, for most objects, IRAS FIR fluxes or upper limits were derived from co-added HCONs.

The spectral energy distributions, from the space-UV to the near-IR, show a slope rising in the UV, or at least flat (in $F_{\nu}$ ). The morphology of the $\mathrm{H} \alpha$ images is very different from that of the continuum. The HII 
regions appear in most cases as bright, concentrated knots on the diffuse continuum images. Some galaxies have a single bright knot, others have a number of HII knots with different brightnesses. The $\mathrm{H} \alpha$ equivalent width is generally very high; e.g. BST324=Mkn49 has an equivalent width of $363 \AA$ at the peak $\mathrm{H} \alpha$ distribution (average equivalent width is $56 \AA$ for the entire galaxy).

The multi-spectral information was used in conjunction with stellar population models (Bruzual and Charlot 1993) to derive the history of star formation for the objects. We found that there was no difference between high HI and low HI galaxies. In all cases, the BCDs must have had a very recent burst of star formation (within the last few $10^{6}$ years). The star-formation rates (SFR) per unit surface of the galaxies, a distanceindependent measurement, range from $2.2810^{-7}$ to $2.7610^{-9} \mathrm{M}_{\odot} /$ year $/ \mathrm{pc}^{2}$. The lower value is comparable with the SFR per unit area of the Galaxy ( $\sim 10^{-9} \mathrm{M}_{\odot} /$ year $/ \mathrm{pc}^{2}$ ).

All objects except one galaxy showed signs of previous star formation, within the last $10^{9}$ years. The one possibly genuine young galaxy, BST144, showed no signatures of an older population. Its $\mathrm{H} \alpha$ equivalent width, for the entire galaxy, is $159 \AA$. No correlation was found with (projected) distance from M87 or the central region of the Virgo cluster and observational parameters connected with star formation.

\section{References}

Bingelli, B., Sandage, A., and Tammann, G.A. (1985) Studies of the Virgo cluster II: A catalog of 2096 galaxies in the Virgo cluster area, Astron. J. Vol. no. 90, pp. 16811758.

Bowyer, S., Sasseen, T.P., Lampton, M. and Wu, X. (1993) In-flight performance and preliminary results from the Far Ultraviolet Space Telescope (FAUST) flown on ATLAS1, Astrophys. J. Vol. no. 415, pp. 875-881.

Bruzual, G. and Charlot, (1993) Spectral evolution of stellar populations using isochrone synthesis, Astrophys. J. Vol. no. 415, pp. 538-553.

Hoffman, G.L., Helou, G., Salpeter, E.E., Glosson, J.F. and Sandage, A. (1987) HI detection survey of a complete magnitude-limited sample of dwarf irregular galaxies in the Virgo cluster area, Astrophys. J. Suppl. Vol. no. 63, pp. 247-264. 